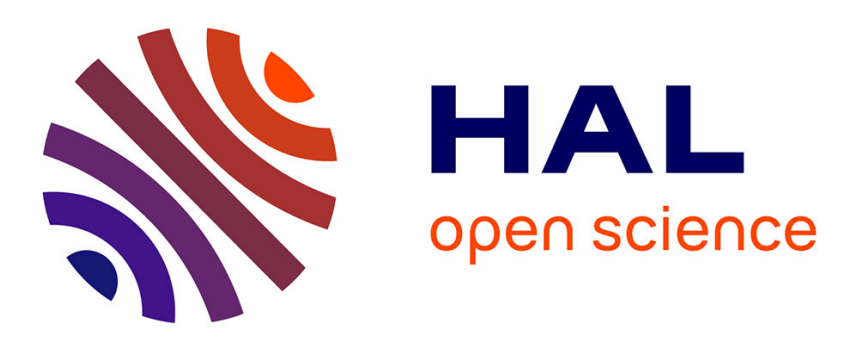

\title{
Quantification of the Local Topological Variations of Stripped and Plated Lithium Metal by X-ray Tomography
}

Lucile Magnier, Didier Devaux, Joël Lachambre, Margaud Lecuyer, Marc Deschamps, Renaud Bouchet, Eric Maire

\section{To cite this version:}

Lucile Magnier, Didier Devaux, Joël Lachambre, Margaud Lecuyer, Marc Deschamps, et al.. Quantification of the Local Topological Variations of Stripped and Plated Lithium Metal by X-ray Tomography. ACS Applied Materials \& Interfaces, 2020, 12 (37), pp.41390-41397. 10.1021/acsami.0c10860 . hal-02946315

\section{HAL Id: hal-02946315 \\ https://hal.science/hal-02946315}

Submitted on 6 Jan 2021

HAL is a multi-disciplinary open access archive for the deposit and dissemination of scientific research documents, whether they are published or not. The documents may come from teaching and research institutions in France or abroad, or from public or private research centers.
L'archive ouverte pluridisciplinaire HAL, est destinée au dépôt et à la diffusion de documents scientifiques de niveau recherche, publiés ou non, émanant des établissements d'enseignement et de recherche français ou étrangers, des laboratoires publics ou privés. 


\title{
Quantification of the local topological
}

\section{variations of stripped and plated lithium metal by X-Ray tomography}

\author{
Lucile Magnier, ${ }^{\dagger, \ddagger}$ Didier Devaux,,${ }^{\ddagger}$ Joël Lachambre, ${ }^{\dagger}$ Margaud Lecuyer, ${ }^{\mathbb{}}$ Marc \\ Deschamps, "Renaud Bouchet, ${ }^{*, \ddagger}$ and Eric Maire ${ }^{*, \dagger}$ \\ †Univ Lyon, INSA Lyon, CNRS UMR 5510, MATEIS, 69621 Villeurbanne, France \\ $\ddagger$ Univ. Grenoble Alpes, Univ. Savoie Mont Blanc, CNRS, Grenoble INP, LEPMI, 38000 \\ Grenoble, France \\ \Blue Solutions, Odet, Ergue Gaberic, 29500 Quimper, France \\ E-mail: renaud.bouchet@grenoble-inp.fr; eric.maire@insa-lyon.fr
}

\begin{abstract}
Lithium ( $\mathrm{Li}$ ) metal is the most promising negative electrode to be implemented in batteries for stationary and electric vehicle applications. For years, its use and subsequent industrialization was hampered because of the inhomogeneous $\mathrm{Li}^{+}$ion reduction upon recharge onto Li metal leading to dendrite growth. The use of solid polymer electrolyte is a solution to mitigate dendrite growth. Li reduction leads typically to dense Li deposits but the Li stripping and plating process remain non-uniform with local current heterogeneities. A precise characterization of the behavior of these heterogeneities during cycling is then essential to move towards an optimized negative electrode. In this work, we have developed a characterization method based on X-ray tomography applied to model Li symmetric cells to quantify and spatially probe the Li stripping/plating
\end{abstract}


processes. Ante- and post-mortem cells are recut in smaller cells in order to allow a $1 \mu m$ voxel size resolution in a conventional laboratory scanner. The reconstructed cell volume is post-processed to numerically re-flatten the Li electrodes, allowing us a subsequent precise measurement of the electrode and electrolyte thicknesses and revealing local interface modifications. This in-depth analysis brings information on the location of heterogeneities and their impact on the electrode microstructure both at the electrode grains and grain boundaries. We show that the plating process (reduction) induces more pronounced heterogeneities compared to the stripping (oxidation) one. The existence of cross-talking between the electrodes is also highlighted. In addition, this simple methodology permits to finely retrieve and then surface-map the local current density at both electrodes based on the local thickness change during the redox process.

\section{Keywords}

lithium metal, X-ray tomography, polymer electrolyte, current density map, lithium microstructure, electrode cross-talking

\section{Introduction}

The current issues related to climate change are leading the society to rethink its transportation modes. ${ }^{1}$ Indeed, oil resources are limited and gasoline vehicles have a high carbon impact. High energy density, long cycle life, and low cost batteries are thus needed to replace the conventional oil based applications. One solution is the development of lithium (Li) metal based batteries. Li metal is the most promising negative electrode thanks to its high theoretical capacity and low electrochemical potential. ${ }^{2,3}$ The development of these batteries has been downplayed due to the heterogeneous Li electrodeposits formed at the Li electrode in combination with conventional liquid-based electrolytes. This process leads 
to the growth of dendrites inducing a short-circuit. ${ }^{4}$ Heterogeneous deposition on the $\mathrm{Li}$ metal surface during battery charge results in low coulombic efficiency, limited cycle life, and safety hazards. ${ }^{5}$ So far, to prevent dendrite growth, the main solutions consist in the addition of electrolyte additives, ${ }^{6-8}$ the anode surface modification, ${ }^{9-12}$ and the replacement of the conventional flammable liquid electrolyte. ${ }^{13-16}$ Theoretically, the use of a non-flammable solid polymer electrolyte, whose shear modulus is sufficiently high, should prevent dendrite growth. ${ }^{17}$ Since the first poly(ethylene oxide)-based polymer electrolyte proposed by Armand et al. ${ }^{18}$ numerous studies have led to the analysis of a wide variety of polymer electrolytes. The combination of both high mechanical resistance and descent ionic conductivity is difficult to obtain ${ }^{19,20}$ but Li metal battery comprising solid polymer electrolyte is commercialy available. ${ }^{21}$ However, characterization and understanding of redox processes at the Li electrode remains essential for battery optimization. Many different techniques are employed to characterize the morphology of the deposits including optical microscopy, ${ }^{22}$ scanning electron microscopy (SEM), ${ }^{23,24}$ atomic force microscopy (AFM) ${ }^{25}$ and Fourier transform infrared spectroscopy (FTIR). ${ }^{26}$ The main disadvantage of these methods is that they are invasive and can deteriorate the probed interfaces during measurement. Non-invasive techniques are thus of interest and among those X-ray tomography combines the advantage of being nonintrusive and particularly well-suited to probe interfaces between soft materials. ${ }^{27,28}$ The group of Balsara et al., have performed synchrotron X-ray tomography on Li symmetric and battery cells comprising a solid polymer electrolyte. They have shown the strong impact of impurities located within the Li bulk on dendrite growth. ${ }^{29-31}$ Using SEM the same group reported that Li grain boundaries control the deposit heterogeneities; the reduction reaction kinetic at grain boundaries is slower than on the crystal faces; reciprocally Li is more easily oxidized from the crystal faces. ${ }^{32}$

In this work, we report on Li plating and stripping processes through a solid polymer electrolyte using model Li symmetric cells. Redox processes have led to dense deposits associated with slight heterogeneous morphologies. We have quantified through a simple methodology 
the Li surface heterogeneities induced by oxidation and reduction reactions by means of Xray tomography performed on a laboratory scanner. Li symmetric cells comprising a solid polymer electrolyte were assembled to characterize both electrodes, anode and cathode, simultaneously and thus both redox processes, i.e. oxidation and reduction. The strong advantage of these model electrochemical cells is the absence of heavy elements usually present in battery cathode materials (Fe, $\mathrm{Ni}, \mathrm{Mn}$, or $\mathrm{Co}$ ). This absence permits to easily optimize the X-ray contrasts and obtain highly defined tomographic images. However, the characterization of complete batteries will be possible using the same analytical method to understand the evolution of Li electrode morphologies, with an optimization of the acquisition parameters or the use of synchrotron sources. We have developed a new image analysis method to spatially resolve preferential reaction zones. The experimental process using post-mortem imaging, the measurements take into account the accumulation of the entire reaction process. Despite the fact that our methodology does not give access to information on the dynamics of redox processes, it has the advantage, contrary to operando measurements, of being representative of phenomena in real batteries (no edge effect due to sample size). In addition, our analysis gives access to quantitative information such as local current density changes, allowing us to compare different cycling parameters (current density, temperature, pressure) on the behavior of the Li heterogeneities.

\section{Results and discussions}

\subsection{Quantification of surface heterogeneity}

All the Li symmetric cells were polarized at a fixed current density $\left(J_{\text {macro }}\right)$ of $0.1 \mathrm{~mA} / \mathrm{cm}^{2}$ in one direction, i.e. one electrode acted as a cathode and the other as an anode. The current was applied for a duration necessary to displace $21.6 \mu \mathrm{m}$ of Li from the anode to the cathode. Prior to the polarization step, the two Li electrodes had an equal thickness. After polarization, the anode thickness decreased while that of the cathode increased. During the 
polarization step, the cell potential $(E)$ was recorded. A typical potential profile, $E$ as a function of the quantity of charge passed $(Q)$, is shown in Figure 1. The potential sharply decreases when the polarization starts. It reaches a minimum at about $1.5 \mathrm{C}$. Then the potential smoothly increases in a linear fashion for higher charge passed values. Such a behavior is typical of a polarization step in Li symmetric comprising liquid- and solid-based electrolytes. ${ }^{33,34}$ During the galvanostatic step, the macroscopic thickness $\left(t_{\text {macro }}\right)$ of the Li stripped from the anode and plated on the cathode is given by the Faraday equation :

$$
t h_{\text {macro }}=\frac{J_{\text {macro }} \times M_{L i}}{F \times \rho_{L i}} \times \Delta t
$$

with $\Delta t$ the polarization time, $M_{L i}$ the Li molar mass, $F$ the Faraday constant, and $\rho_{L i}$ the Li density.

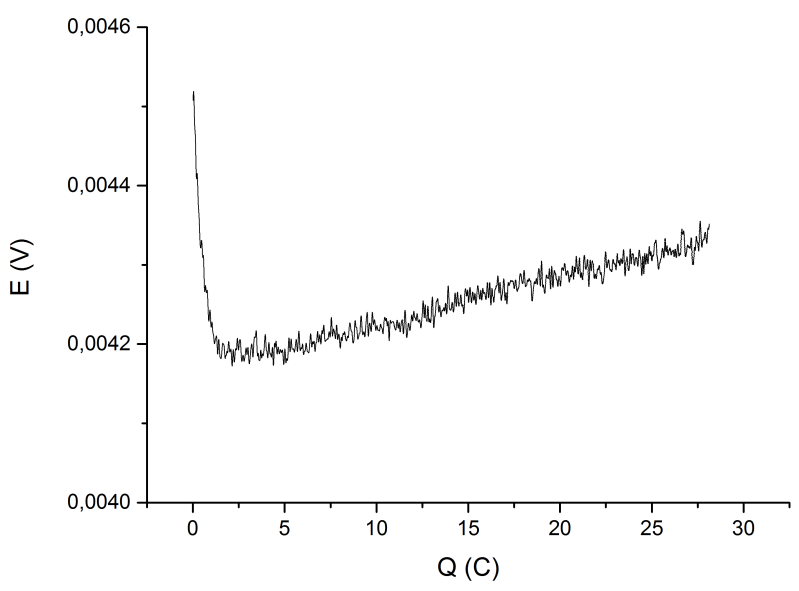

Figure 1: Typical evolution of the Li symmetric cell potential $(E)$ as a function of the charge passed $(Q)$.

After the electrochemical step, the Li symmetric cells were opened in an Argon glove box and a portion of the cells located in its middle was cut out. This cell portion was placed in a sealed polypropylene tube (a $5 \mathrm{~mm}$ diameter tube) and then mounted on a laboratory X-ray tomography scanner to be imaged at room temperature. After computing reconstruction, a 3D volume of the illuminated sample was obtained. Typical tomographic slices, along a $(\mathrm{x}, \mathrm{z})$ plane, of an uncycled and a cycled cell obtained after 3D reconstruction are presented 
in Figures 2.a and c. The pixel size was set to $1 \mu \mathrm{m}$. At a specific position in the (x,z) coordinates the gray value of a pixel is related to the linear X-ray absorption coefficient that mostly depends on the electron density of the considered material. Li, a lightweight element (atomic number 3), is a low X-rays absorbent material and appears dark gray in Figures 2.a and c, while oxygen- and carbon-rich electrolyte material appears brighter. The thickness of the electrolyte is about $14 \mu \mathrm{m}$. The resolution of $1 \mu \mathrm{m}$ is then more than enough to visualize this layer. The electrolyte absorbs X-rays much more than the Li so the contrast in the images is due to attenuation contrasts.

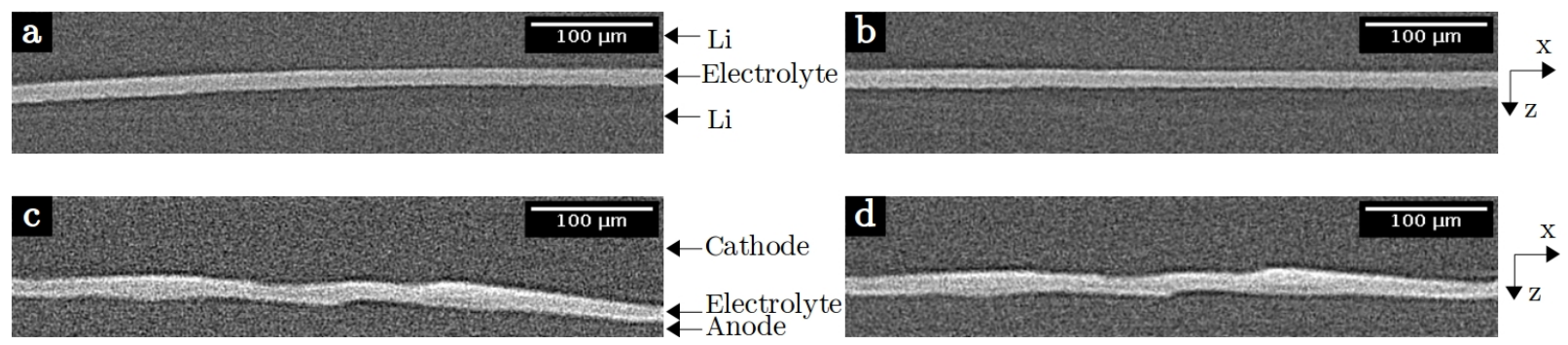

Figure 2: Typical X-ray tomography images (a) uncycled cell, (b) after flattening of the uncycled cell, (c) cycled cell, (d) after flattening of the cycled cell.

Because of the softness of the Li electrodes and flexibility of the polymer electrolyte, the preparation of the sample for tomography analysis often induces a slight curvature of the samples. This effect is clearly seen in Figures 2.a and $\mathrm{c}$ in which the polymer electrolyte layer is bended. To correct for this, as a first step, all the tomographic images were numerically processed using a Python routine developed aiming at flattening the interfaces. This method is relevant if two hypotheses are respected: (i) the average curvature of the film is small; (ii) the film thickness is small with respect to the radius of curvature. In short, one of the curved Li surfaces in contact with the argon atmosphere inside the polypropylene tube is taken as a reference surface for the Python routine (corresponding to the surface of Li opposite the Li-electrolyte interface). Note that these surfaces are out of the field of view in Figures 2.a and $\mathrm{c}$ as these figures show a zoom around the electrolyte. The $\mathrm{z}$ positions of each voxel composing this surface are recorded and placed in a new $(\mathrm{x}, \mathrm{y})$ planar surface. The selection 
of this surface is made by segmentation of the Li/argon interface. This step is crucial and selection errors can locally induce important defects on the surface. It is therefore necessary to smooth the surface (by a low pass filter) to satisfy hypothesis (i) (see scheme on Supporting information Figure S1). A distance map to the curved reference surface is then calculated. The numerical flattening process consists in considering the reference surface as perfectly flat, i.e. each $(\mathrm{x}, \mathrm{y})$ position has identical $\mathrm{z}$ coordinate. Then the coordinates $(\mathrm{x}, \mathrm{y}, \mathrm{z})$ of each other voxels are recalculated to preserve their distance from the initial reference surface along a normal to this surface. The result of this numerical flattening process is a new stack of tomograms made of flat electrodes. The result of this process is shown in Figures 2.b and $\mathrm{d}$ which correspond to the tomograms shown in Figures 2.a and c, respectively, after application of the numerical flattening process. The main interest of this routine is that whilst the Li electrodes are both flats, the two interfaces in between the Li electrode and the solid polymer electrolyte that contain some defects (Figure 2.c) are preserved by the flattening routine (Figure 2.d).
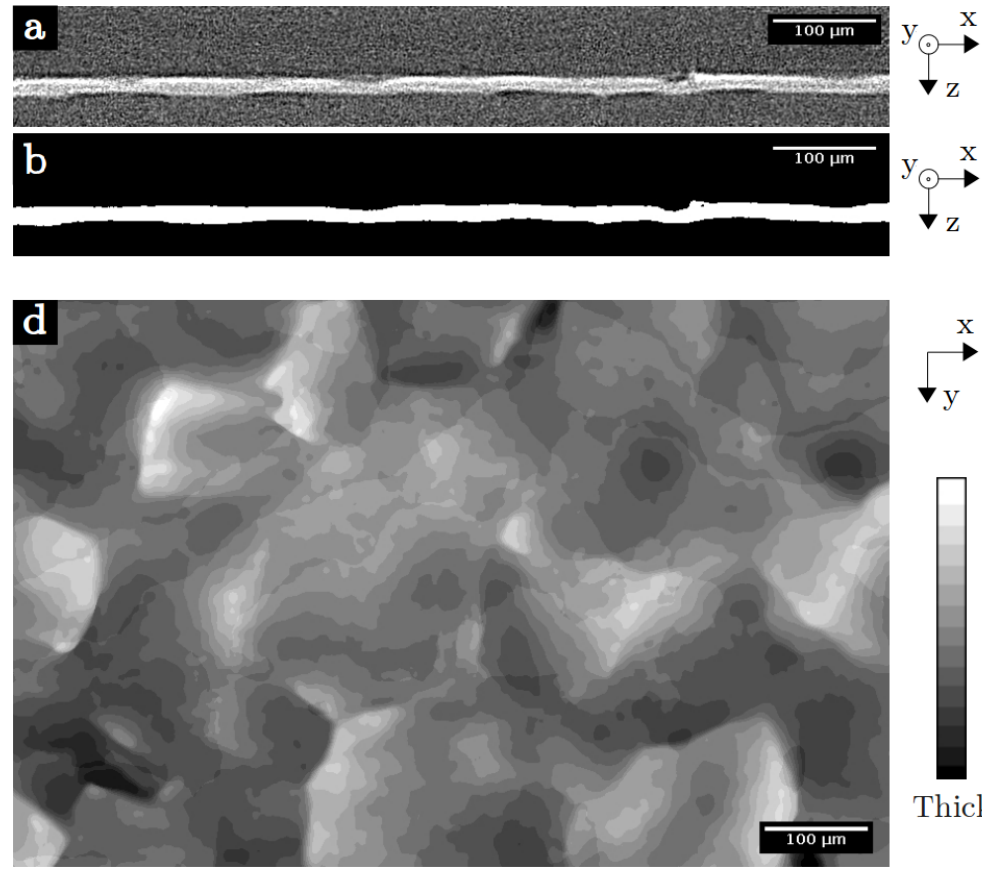

Z project

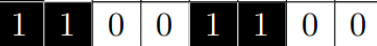

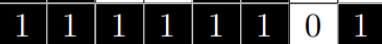

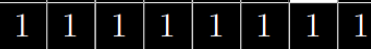
\begin{tabular}{l|l|l|l|l|l|l|l|}
\hline 1 & 1 & 1 & 1 & 1 & 1 & 1 & 1
\end{tabular}
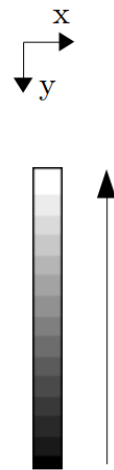

Thickness

Figure 3: Image segmentation and process of the X-Ray tomogram (a) X-ray absoprtion tomography image, (b) thresholded binarized image, (c) thickness measurement principle, (d) 2D map of the cathode thickness in arbitrary unit. 
The second step in the X-ray image analysis is the segmentation of the electrodes. By adjusting a threshold gray value, the voxels corresponding to the Li electrodes can be separated from those of the polymer electrolyte and the argon. Manual cleaning eliminates segmentation errors. In the literature, a similar routine was proven effective on $\mathrm{Li}$ symmetric and $\mathrm{Li}$ metal based batteries. ${ }^{30,31}$ As an example, Figure 3.a shows a typical tomographic image of a Li symmetric cell. The segmentation process leads to a binary image: Figure 3.b shows the binarized version of Figure 3.a in which the color of the Li electrodes and the electrolyte are black and white, respectively.

The third step consists in the calculation of the thicknesses of each electrode. On the binary images (see Figure 3.b) at each black voxel the arbitrary value of 1 is assigned and all the white voxels are set to 0 as shown schematically in Figure 3.c. By summing the voxel values in each column along the $\mathrm{z}$ axis, we obtain for each $(\mathrm{x}, \mathrm{y})$ coordinate the number of black voxels. The experimental isotropic voxel size being equal to $1 \mu m$ for our X-ray tomography setup, the sum of the voxels according to $\mathrm{z}$ is directly the electrode thickness (see the last line in Figure 3.c). This tomographic image analysis produces a 2D topological map in the $(\mathrm{x}, \mathrm{y})$ plane for both Li electrodes for which each pixel contains the local electrode thickness value. Those pixels can then be scaled in gray, or color, values to highlight the presence of local thickness changes. An example of a 2D topological map, scaled in gray values, of a cathode electrode after cell polarization is shown in Figure 3.d. In this figure the white and black pixels correspond to the highest and lowest thickness areas, respectively.
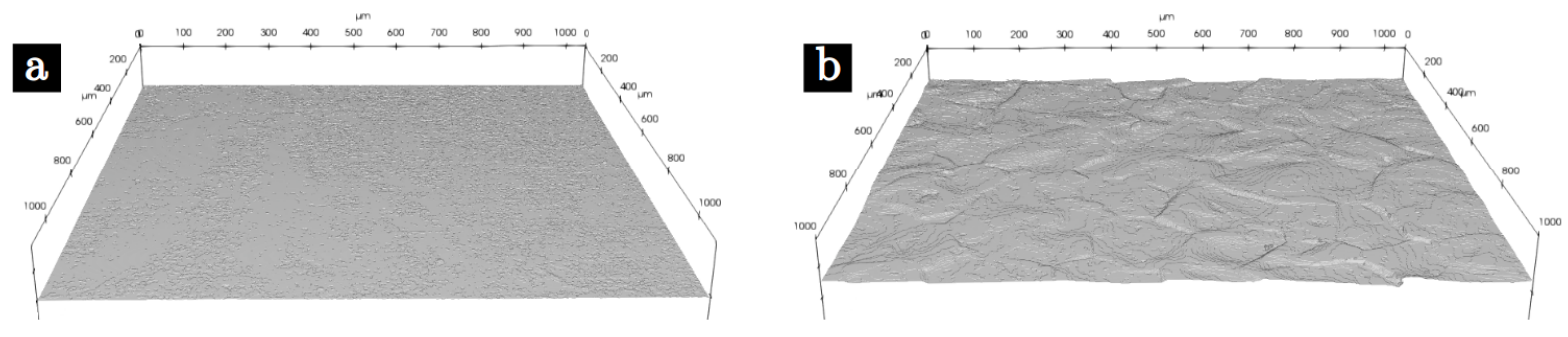

Figure 4: 3D visualization of the Li surface: (a) initial state (fresh cell), (b) final state (cycled cell).

To go further and conclude on the cause of the local thickness variation observed in Figure 
3.d, the initial state of the Li surface must be determined on a fresh cell, i.e. uncycled Li symmetric cell. Our methodology being post-mortem by nature, the initial state of Li surface electrodes facing the polymer electrolyte was determined on a $\mathrm{Li}$ symmetric cell replicate placed under similar pressure and temperature to match the initial state of the cycled cell. 3D global overviews of the scanned electrode surface after flattening and segmentation are displayed in Figure 4.a and b for the uncycled and cycled Li surface, respectively. The contrasts in this figure reflect the roughness of the Li/electrolyte interface with an arbitrary scale. In Figure 4.a, the initial morphology of the Li electrodes has very little variations in thickness which are at the limit of the measurement sensitivity $(\sim 1 \mu m)$. These variations are much smaller than the fluctuations measured on cycled cells (Figure 4.b). In addition, the grain boundaries of the uncycled electrodes are not revealed. Since the electrodes are initially rather flat, we thus attribute the local thickness variations on the cycled cell (Figure 3.d) to the impact of the redox processes. In Figure 3.d, the regions of small thicknesses (black pixels) are thus attributed to area where lesser Li is reduced. Conversely, regions of large thicknesses (white pixels) are areas with a stronger reduction. In summary, through the methodology presented here, the topology of the rendered surface permits to finely track the heterogeneity in the stripping and plating electrochemical processes at the Li/electrolyte interfaces. In all the tomographic slices showed in Figures 2 and 3, dark/bright fringes can be seen at each interface. These are typical "phase contrast" fringes, very frequent in highly coherent synchrotron X-Ray sources, ${ }^{35}$ and also present in our case because of the very small size of our focal point.

The Faraday's law (see Equation 1) can allow us to establish a direct theoretical relationship between the thickness of Li displaced and the current density. We can suppose that it remains valid at both macroscopic (cell) and microscopic (local) scale. We can then in principle directly convert local thickness changes $\left(\Delta t h_{x, y}\right)$ into local current density variations $\left(\Delta J_{x, y}\right)$. The local thickness change at a specific $(\mathrm{x}, \mathrm{y})$ pixel is calculated as the difference between the initial thickness of the electrode and the local thickness extracted on the tomography 

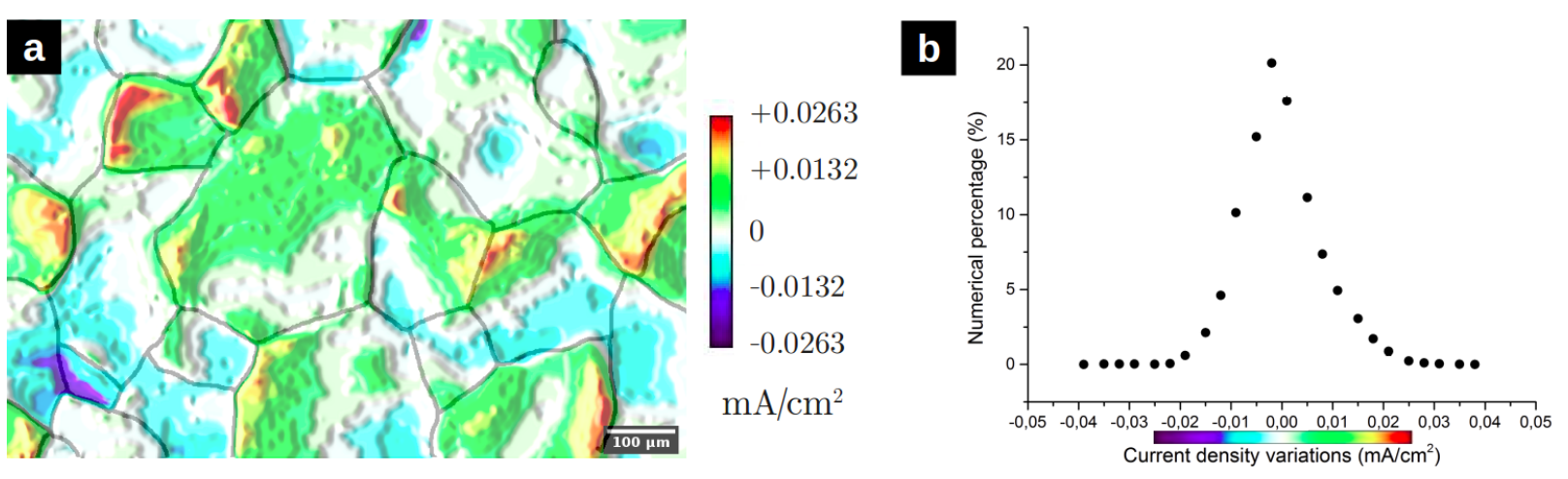

Figure 5: (a) 2D map of local current density variations overlaped with Li grain boundaries, (b) histogram of variations extracted from the map.

image using our mapping procedure (see Figure 3). The initial thickness has been measured macroscopically prior to the assembly of the cell. It is assumed to be constant in every location $(\mathrm{x}, \mathrm{y})$ of the cell. The calculation of the average between the anode and cathode thickness is similar to the initial thickness. Lateral effects are then negligible at the scale of the $2 \mathrm{D}$ map. Equation 2 is then used in a straightforward manner to link the macroscopic and the local scale:

$$
\frac{J_{\text {macro }}}{t h_{\text {macro }}}=\frac{\Delta J_{x, y}}{\Delta t h_{x, y}}=\frac{F \times \rho_{L i}}{M_{L i} \times \Delta t}
$$

Using Equation 2, the map of cathode thickness (see Figure 3.d) can be converted into a local current density variations map as shown in Figure 5.a. The color scale in this figure is the variations of the local current density with respect to the average current density, i.e. $\Delta J_{x, y}$. The white areas correspond to locations where the Faraday's law is exactly that obtained at the macroscopic scale. Favored reduction sites where the local current densities are higher appear as a gradient ranging from green up to red. Conversely, weakly reduced areas, corresponding to small thicknesses are colorized by a gradient from blue to purple and were therefore subjected to local current densities lower than the average. This detailed image processing provides information about the location of Li deposits. In addition, to gather qualitative information on the location of Li deposits, the image analysis can also give quantitative information. Figure 5.b shows the histograms of the variations calculated on the map of Figure 5.a. The standard deviation $(S D)$ of this distribution can then be 
calculated. In the case of this cathode, $S D$ of the local current density variations is 0.0078 $m A / \mathrm{cm}^{2}$.

To tackle the issue of the non-homogeneous redox processes at stake in the Li metal, the local variations in thicknesses, or current densities, can be compared with the Li microstructure. The pattern of the grain boundaries can also be extracted from the X-ray tomography stack, as exemplified in Figure S2, where the grains are clearly visible at Li/electrolyte interface by a human's eye. Automatic detection of these grains is impossible however so their outlining has to be performed manually. In Figure 5.a such a manually outlined pattern is overlapped with the local current density variations map. We observe that the grains and grain boundaries have a minor correlation with the electroactivity of the Li electrode. Some grains are more reduced than others. In a given grain, the reduction process can also be spatially non homogeneous. The reasons for this behavior is outside the scope of this paper and will be analyzed further in the future. The use of a Li metal electrode requires consideration of its reactivity with the electrolyte. To the best of our knowledge, all of the electrolytes, from conventional liquid to the polymer as PEO, naturally reacts when in contact with Li metal generating the so-called SEI (Solid Electrolyte Interphase) layer. ${ }^{36}$ This SEI layer is known to play an important role on the Coulombic efficiency and morphology of the electrodeposits. However, after the initial SEI formation, ${ }^{37}$ PEO based electrolytes are electrochemically stable towards Li metal as reported by cyclic voltammetry experiments at the low potential of $\mathrm{Li}^{+} / \mathrm{Li}^{0}$ and by the cycling of a $\mathrm{Li} / \mathrm{LiFePO}$ battery for more than 600 cycles associated with a very low capacity fading $(\sim 0.02 \%$ per cycle $)$ and a faradic efficiency of $99.98 \%{ }^{38}$ Looking at interfacial reactions to characterize the few nanometer thick SEI layer would require a chemically-sensitive surface technique (XPS). So far at synchrotron, X-ray nanotomography is only able to go down to a $50 \mathrm{~nm}$ voxel size which is still too large to get insight on the interfacial Li/electrolyte reactions. This new image analysis method allows us the visualization of preferential electrochemical activity zones and leads to a fundamental understanding of the consequences of electrochemical processes on the surface 
morphology of Li metal electrodes. It also gives direct access to quantitative data that makes it possible to compare different parameters (temperature, current density,...) as well as the direct comparison of local thickness variation of two electrodes (anode and cathode) facing each other.

\subsection{Comparison of the heterogeneities induced by oxidation and reduction}

It is possible to apply our new analysis method to the complete cell after polarization. We can thus compare the impact of oxidation and reduction. Figure 6.a shows the map of the local current density variations of the anode relative to the average current density. It should be noted that in the case of the anode, the high local current densities correspond to small electrode thicknesses after polarization. Indeed, these zones correspond to regions where a larger quantity of $\mathrm{Li}$ was oxidized. For the cathode, the high local current densities are the high electrode thicknesses after polarization (larger quantity of Li reduced). Figure 6.b shows the result of a similar analysis performed on the cathode side of the same cell. Both electrodes, anode and cathode, display thickness variations showing that reduction and oxidation are spatially heterogeneous processes. The outline of the grains is superimposed on both local current density variations maps in Figures 6.a and b. For the electrochemical cells studied here, the comparison of the local current density variations and the Li microstructures show that the redox processes do not occur at particular locations, i.e. inside grains and/or at grain boundaries. This result does not correlate the observations made by the group of Balsara et al., where Li reduction at grain boundaries was slower than on the crystal faces and Li oxidation faster on crystal faces. It should be noted that the group of Balsara et al. was using a different solid polymer electrolyte and that their SEM observations were only

qualitative. ${ }^{32}$ In addition, we believe that this non-correlation can also be explained by the difference in Li origin and especially, its microstructure in terms of grains, precipitates and inclusions. Further investigations are needed to fully understand this behavior. 

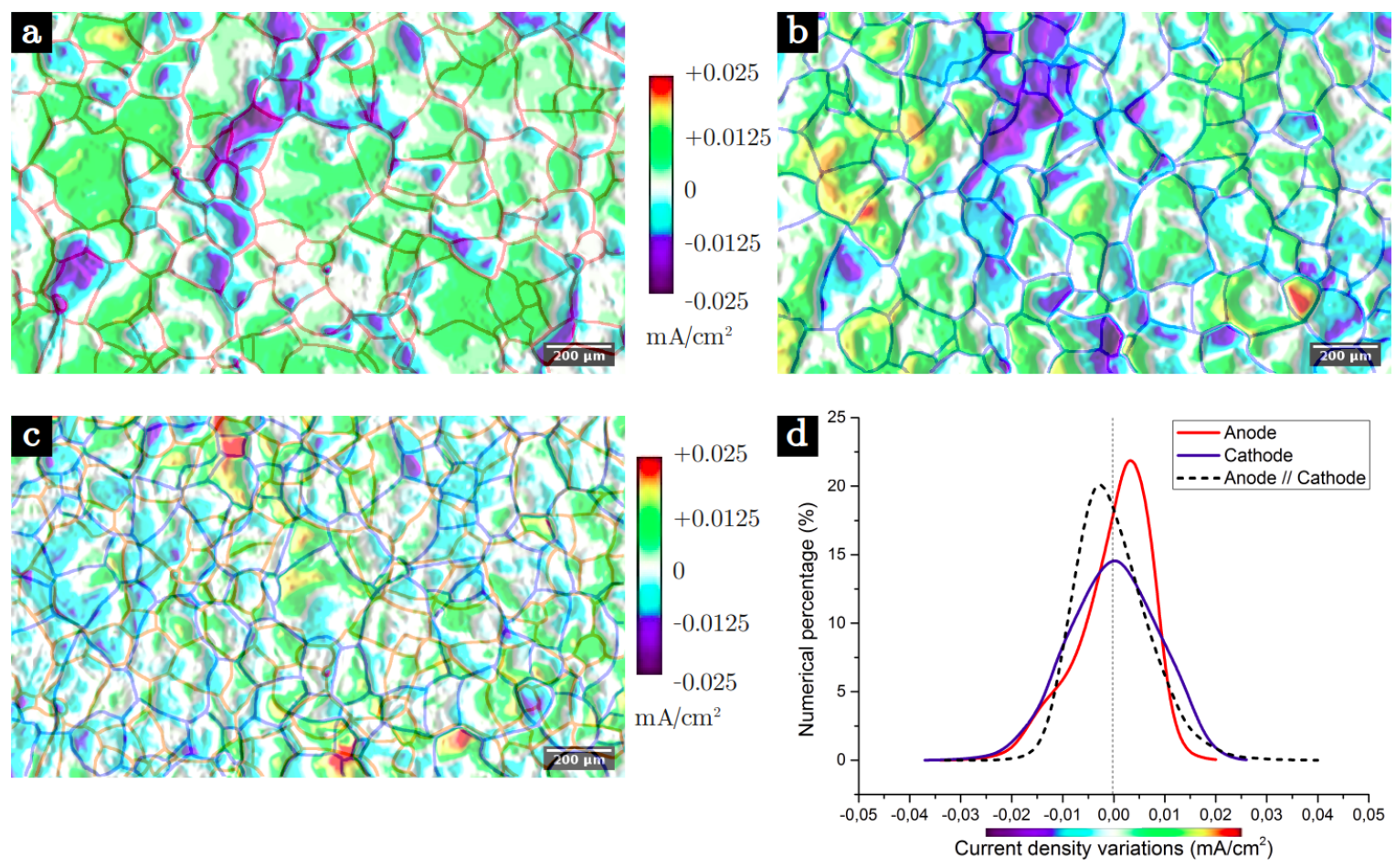

Figure 6: 2D map of local current density variations for (a) the anode with grain boundaries pattern in red, (b) the cathode with grain boundaries pattern in blue; (c) highlight of crosstalking anode//cathode (subtraction of the local current densities of the anode (Figure 6.a) by those of the cathode (Figure 6.b)) with grain boundaries patterns of the anode (red) and the cathode (blue); (d) histogram of local current density variations.

The distributions of local current density variations are given in Figure 6.d, the red line corresponds to the anode and the blue line to the cathode. For both electrodes, statistical data such as minimal value (Min value), maximal value (Max value), full-width-at-half-maximum (FWHM), and standard deviation $(S D)$ can be extracted from these histograms (Figure 6.d) and are reported in Table 1. The cathode has a lower Min value and a higher Max value. This means that the reduction leads to more extreme local current densities. The cathode has also a higher $S D$ and a higher $F W H M$. In terms of surface area, the cathode is therefore more subject to current densities far from the average than the anode. The different statistical parameters all demonstrate the presence of more heterogeneities during the reduction process. The analysis presented here corresponds to one cell but the analysis of 9 other cells leads to similar conclusions; reduction leads to more thickness heterogeneities than oxidation. 
Table 1: Statistical values extracted from the histogram (Figure 6.d).

\begin{tabular}{cccc}
\multicolumn{4}{c}{ Local current density variations $\left(\mathrm{mA} / \mathrm{cm}^{2}\right)$} \\
\hline & Anode & Cathode & Anode//Cathode \\
\hline Min value & -0.0340 & -0.0370 & -0.0330 \\
Max value & 0.0200 & 0.0260 & 0.0400 \\
FWHM & 0.0125 & 0.0228 & 0.0165 \\
SD & 0.0071 & 0.0087 & 0.0071 \\
\hline
\end{tabular}

Another feature that can be extracted from this image analysis is the cross-talking between the Li electrodes. Figure 6.c corresponds to the subtraction of the local current densities of the anode by that of the cathode. This anode//cathode mapping directly shows the link between the anode and the cathode. On the one hand, in Figure 6.c, the red $(+0.025$ $\left.\mathrm{mA} / \mathrm{cm}^{2}\right)$ and purple $\left(-0.025 \mathrm{~mA} / \mathrm{cm}^{2}\right)$ zones are the zones of strong fluctuations between anode and cathode. In these areas, the cross-talking between the two electrodes is weak: the morphology caused by the oxidation of $\mathrm{Li}$ is independent of that caused by the reduction. On the other hand, in the white areas, the difference in current density between the anode and the cathode is small (close to $0 \mathrm{~mA} / \mathrm{cm}^{2}$ ). These are areas where the places of oxidation and reduction are strongly linked. In Figure 6.c, we can therefore see that despite the presence of a few red and purple areas, a large part of the surface analyzed is close to a fluctuation around $0 \mathrm{~mA} / \mathrm{cm}^{2}$. This is an illustration of the existence of cross-talking between the anode and the cathode. Grain boundaries patterns of the anode (red) and the cathode (blue) are superimposed to the local current density variations of anode//cathode. At this stage of our work, the impact of anode and cathode microstructure on cross-talking location is yet to be determined.

The proof of the existence of this cross-talking can be brought by the comparison of the histograms of local current densities. The distribution of local current densities of Figure 6.c is represented by the black dash line in Figure 6.d. In the same way as above, it is possible to extract statistical parameters from it (see the Anode//Cathode column in Table 1). The anode//cathode mapping is characterized by a Min value closer to $0 \mathrm{~mA} / \mathrm{cm}^{2}$ than the anode and the cathode as well as by a lower $S D$ than that of the cathode and equal to that of the 
anode. These two parameters indicate that the anode//cathode parallel is less heterogeneous than the anode and the cathode taken separately. If there was no correlation between the electrodes, the heterogeneities should be amplified and therefore the $S D$ should be twice that of the electrodes alone. This proves the existence of cross-talking: the variations in surface morphology created by oxidation and reduction are interdependent. The $F W H M$ value is between that of the anode and the cathode. This also seems to indicate the interdependence between the two electrodes since the combination of the two electrodes generally minimizes large variations in current densities. Only the Max value of the anode//cathode distribution is greater than the anode and the cathode taken separately. The cross-talking between the two electrodes is therefore not perfect over the entire surface and, in certain areas, oxidation and reduction are independent. The fact that this non-symmetry between the two electrodes can only be quantified by the Max value indicates that this is an occasional phenomenon. On most of the surface, cross-talking exists between the two electrodes.

\section{Conclusion}

The use of a solid polymer electrolyte leads to dense but irregular Li deposits upon cycling. Indeed, small heterogeneities at the Li surface are induced by both oxidation and reduction processes. Until now, their characterization was only qualitative. Our research focused on the quantification of the Li surface heterogeneities after oxidation and reduction, using symmetric Li-electrolyte-Li cells. We put these cells under a constant polarization in order to move Li from the anode to the cathode. Our characterization technique consisted in X-ray imaging in order to visualize interfaces in a non-intrusive way without modifying the fragile interfaces of interest during sample preparation. Unlike SEM or AFM, X-ray tomography allows us to characterize the microstructure in a "quasi non-destructive" way and in 3D, which would be difficult with other methods. We have developed an image analysis method based on the creation of 2D mapping of electrode thicknesses. These maps could be trans- 
formed into local current density variations, and directly give information on the location of the preferential zones of oxidation and reduction. Our analysis method also gives access to quantitative information on the thickness heterogeneities. The comparison of different parameters is thus made possible. We have compared the impact of oxidation and reduction. We have shown that both processes lead to heterogeneities of electrode thicknesses. These two electrochemical processes are therefore governed by variations in local current densities. In both cases, the microstructure of $\mathrm{Li}$ is correlated with heterogeneities. Some grains are more electrochemically active than others. The reasons for this behavior are still unknown but will be the subject of future studies. We have also shown through the quantitative comparison of the two electrodes that the reduction leads to more important heterogeneities than the oxidation. This study has also highlighted the existence of cross-talking between the electrodes showing a correlation between the places of oxidation and reduction. Our future work will consist in the study of other external parameters that can modify the behavior of Li in oxidation and reduction, as well as in the study of the impact of cycling on the morphology of $\mathrm{Li}$ electrodes. We believe that this type characterization will bring fundamental understanding on Li behavior.

\section{Experimental section}

\subsection{Sample preparation}

Li symmetric cells comprising a solid polymer electrolyte were assembled in an Argon filled glove box (Jacomex). There, a Li metal foil (of $60 \pm 5 \mu m$ thick) was pressed onto a poly(ethylene oxide)-based solid electrolyte layer (of $14 \mu m$ thick) then laminated to ensure good adhesion. A second Li foil was added onto the electrolyte side before a second laminating step. Copper current collectors were then attached on both Li electrode edges. The resulting assembly is shown in Figure 7.a. The Li symmetric cell was placed in a pouch 

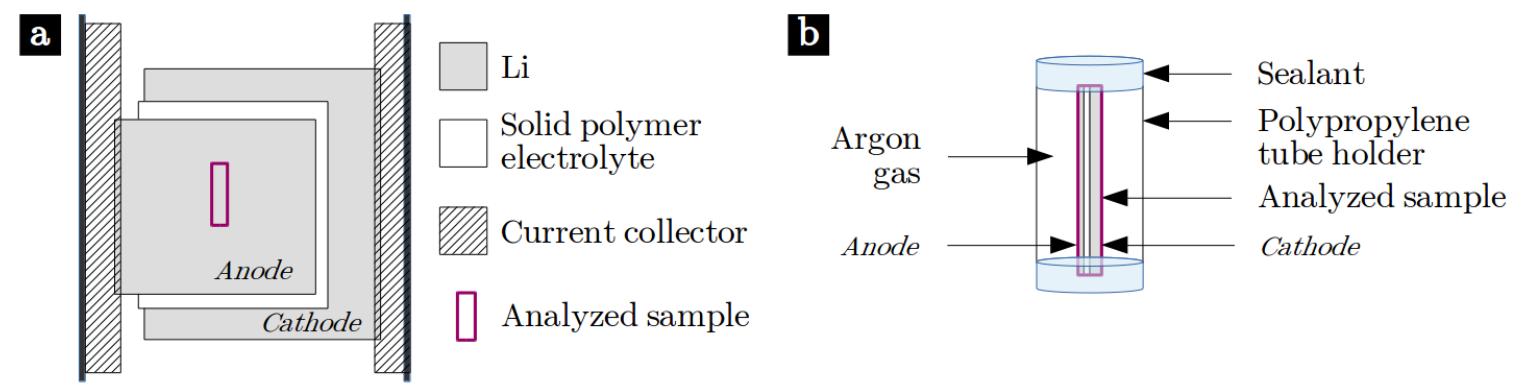

Figure 7: (a) Li symmetric cell assembly ; (b) schematic representation of X-ray tomography sample preparation

bag and vacuum sealed. Before cycling, the Li symmetric cells were placed in a compression home-made cell holder applying a constant external pressure of 2 bars. Each cell were placed in an oven (Memmert) held at a temperature of $80^{\circ} \mathrm{C}$ and the current collectors were connected to a VMP3 potentiostat (BioLogic SAS). The Li symmetric cells were galvanostatically polarized at a constant current density of $0.1 \mathrm{~mA} / \mathrm{cm}^{2}$ in order to move a $21.6 \mu \mathrm{m}$ layer of Li from the anode to the cathode side. To study the initial state, the cell was placed under pressure and temperature for several days without applying any current before being analyzed. After polarization, the cells were taken out of the holder and transferred back to the glove box to be removed from the pouch bag. A rectangle, typically of $3 \mathrm{~mm}$ per 10 $\mathrm{mm}$, was cut at the center of the cell and gently placed in a polypropylene tube holder (a 5 $\mathrm{mm}$ diameter tube). Both extremities of the tube were then sealed using epoxy resin that was dried for a sufficient amount of time in the glove box. To avoid sample motion during the acquisition, the two short edges of the cutted sample are glued with the resin sealing the tube (see Figure 7.b). This operation is performed in the Argon filled glove box. All the tubes were stored safely inside the glove box. When necessary, the samples were taken out of the glove box and transferred to a laboratory X-ray tomograph (EasyTom, RX Solutions).

\subsection{X-Ray tomography imaging}

All the sample were imaged at room temperature. To ensure a vertical position of the sample, the sample-containing tube was glued to an alumina stem. This stem was itself 
fixed to the rotation platform of the tomograph. A tension of $50 \mathrm{kV}$ was used to acquire the scan with a voxel size of $1 \mu \mathrm{m}$. The acquisition was made with a continuous mode recording 4000 images for a $360^{\circ}$ rotation. An exposure time of $0.60 \mathrm{~s}$ provided enough X-ray flux to ensure a good acquisition of the radiographs. The active surface area of $1880 \mu \mathrm{m} \times 1120 \mu \mathrm{m}$ was imaged in $40 \mathrm{~min}$. The collected radiographs were reconstructed using the commercial software provided by RX solutions to produce a 3D stack. The contrast in the images is due to attenuation contrast. The attenuation contrast is very small in both $\mathrm{Li}$ and PEO at 50 $\mathrm{kV}$ but the difference between the two materials can still easily be made (see the difference in gray level in Figure 2). Further image analysis was performed using the Fiji shareware. ${ }^{39}$

\section{Supporting Information Available}

Scheme of the condition necessary for flattening (Figure S1) ; Grain boundaries visualization (Figure S2).

\section{Author Contributions}

LM assembled and tested the cells. DD and RB assisted in the interpretation of the electrochemical data. LM acquired and analyzed the X-ray tomography data. JL implemented python code to flatten the electrodes. EM assisted in the interpretation and image proccessing of the tomograms. DD, ML, MD, RB and EM helped discuss the results. RB and EM conceived the study. LM,DD,EM,RB wrote the manuscript, all the authors participated to the preparation of the manuscript. 


\section{Acknowledgments}

Financial support by Blue Solutions compagny (CIFRE convention n²017/0574).

\section{References}

(1) Chapman, L. Transport and Climate Change: a Review. Journal of Transport Geography 2007, 15, 354-367.

(2) Tarascon, J.-M.; Armand, M. Issues and Challenges facing Rechargeable Lithium Batteries. Nature 2001, 414, 359-367.

(3) Xu, W.; Wang, J.; Ding, F.; Chen, X.; Nasybulin, E.; Zhang, Y.; Zhang, J.-G. Lithium Metal Anodes for Rechargeable Batteries. Energy Environ. Sci. 2014, 7, 513-537.

(4) Rosso, M.; Brissot, C.; Teyssot, A.; Dolle, M.; Sannier, L.; Tarascon, J.-M.; Bouchet, R.; Lascaud, S. Dendrite Short-Circuit and Fuse Effect on Li/Polymer/Li Cells. Electrochimica Acta 2006, 51, 5334-5340.

(5) Aurbach, D.; Zinigrad, E.; Teller, H.; Dan, P. Factors Which Limit the Cycle Life of Rechargeable Lithium (Metal) Batteries. Journal of The Electrochemical Society 2000, $147,1274-1279$.

(6) Liu, S.; Li, G.-R.; Gao, X.-P. Lanthanum Nitrate As Electrolyte Additive To Stabilize the Surface Morphology of Lithium Anode for Lithium-Sulfur Battery. ACS Applied Materials \& Interfaces 2016, 8, 7783-7789.

(7) Kuwata, H.; Sonoki, H.; Matsui, M.; Matsuda, Y.; Imanishi, N. Surface Layer and Morphology of Lithium Metal Electrodes. Electrochemistry 2016, 84, 854-860. 
(8) Zheng, J.; Engelhard, M. H.; Mei, D.; Jiao, S.; Polzin, B. J.; Zhang, J.-G.; Xu, W. Electrolyte Additive Enabled Fast Charging and Stable Cycling Lithium Metal Batteries. Nature Energy 2017, 2, 1-8.

(9) Hwang, S. W.; Yom, J. H.; Cho, S. M.; Yoon, W. Y. Electrochemical Behavior of Li$\mathrm{Cu}$ Composite Powder Electrodes in Lithium Metal Secondary Batteries. ACS Applied Materials \& Interfaces 2017, 9, 22530-22538.

(10) Yang, W.; Yang, W.; Sun, B.; Di, S.; Yan, K.; Wang, G.; Shao, G. Mixed Lithium Oxynitride/Oxysulfide as an Interphase Protective Layer To Stabilize Lithium Anodes for High-Performance Lithium-Sulfur Batteries. ACS Applied Materials $\mathcal{G}$ Interfaces 2018, 10, 39695-39704.

(11) Lu, Z.; Zhang, Z.; Chen, X.; Chen, Q.; Ren, F.; Wang, M.; Wu, S.; Peng, Z.; Wang, D.; Ye, J. Improving Li Anode Performance by a Porous 3D Carbon Paper Host with Plasma Assisted Sponge Carbon Coating. Energy Storage Materials 2018, 11, 47-56.

(12) Matsuda, S.; Kubo, Y.; Uosaki, K.; Nakanishi, S. Lithium-Metal Deposition/Dissolution within Internal Space of CNT 3D Matrix Results in Prolonged Cycle of Lithium-Metal Negative Electrode. Carbon 2017, 119, 119-123.

(13) Tsao, C.-H.; Hsiao, Y.-H.; Hsu, C.-H.; Kuo, P.-L. Stable Lithium Deposition Generated from Ceramic-Cross-Linked Gel Polymer Electrolytes for Lithium Anode. ACS Applied Materials \& Interfaces 2016, 8, 15216-15224.

(14) Baik, J.-H.; Kim, S.; Hong, D. G.; Lee, J.-C. Gel Polymer Electrolytes Based on Polymerizable Lithium Salt and Poly(ethylene glycol) for Lithium Battery Applications. ACS Applied Materials \&5 Interfaces 2019, 11, 29718-29724.

(15) Choudhury, S.; Stalin, S.; Vu, D.; Warren, A.; Deng, Y.; Biswal, P.; Archer, L. A. Solid-State Polymer Electrolytes for High-Performance Lithium Metal Batteries. Nature Communications 2019, 10, 1-8. 
(16) Shin, J.-H.; Henderson, W. A.; Passerini, S. PEO-Based Polymer Electrolytes with Ionic Liquids and Their Use in Lithium Metal-Polymer Electrolyte Batteries. Journal of The Electrochemical Society 2005, 152, A978-A983.

(17) Monroe, C.; Newman, J. The Impact of Elastic Deformation on Deposition Kinetics at Lithium/Polymer Interfaces. Journal of The Electrochemical Society 2005, 152, A396A404.

(18) Armand, M.; Chabagno, J.; Duclot, M. Fast Ion Transport in Solids: Electrodes and Electrolytes; Ed. P. Vashishta, J. N. Mundy, North-Holland: Amsterdam, 1979.

(19) Armand, M. Polymer Solid Electrolytes - an Overview. Solid State Ionics 1983, 9-10, $745-754$.

(20) Manuel Stephan, A.; Nahm, K. Review on Composite Polymer Electrolytes for Lithium Batteries. Polymer 2006, 47, 5952-5964.

(21) Autolib' : la voiture electrique en libre-service debarque a Paris, [Online], Available: https://www.lemonde.fr/societe/article/2011/10/02/autolib-la-voiture-electrique-enlibre-service-debarque-a-paris_1581177_3224.html. 2011,

(22) Lane, G. H.; Best, A. S.; MacFarlane, D. R.; Forsyth, M.; Hollenkamp, A. F. On the Role of Cyclic Unsaturated Additives on the Behaviour of Lithium Metal Electrodes in Ionic Liquid Electrolytes. Electrochimica Acta 2010, 55, 2210-2215.

(23) Gireaud, L.; Grugeon, S.; Laruelle, S.; Yrieix, B.; Tarascon, J.-M. Lithium Metal Stripping/Plating Mechanisms Studies: A Metallurgical Approach. Electrochemistry Communications 2006, 8, 1639-1649.

(24) Lopez, C. M.; Vaughey, J. T.; Dees, D. W. Morphological Transitions on Lithium Metal Anodes. Journal of The Electrochemical Society 2009, 156, A726-A729. 
(25) Morigaki, K.-i.; Ohta, A. Analysis of the Surface of Lithium in Organic Electrolyte by Atomic Force Microscopy, Fourier Transform Infrared Spectroscopy and Scanning Auger Electron Microscopy. Journal of Power Sources 1998, 76, 159-166.

(26) Morigaki, K.-i. Analysis of the Interface Between Lithium and Organic Electrolyte Solution. Journal of Power Sources 2002, 104, 13-23.

(27) Frisco, S.; Liu, D. X.; Kumar, A.; Whitacre, J. F.; Love, C. T.; Swider-Lyons, K. E.; Litster, S. Internal Morphologies of Cycled Li-Metal Electrodes Investigated by NanoScale Resolution X-ray Computed Tomography. ACS Applied Materials $\mathcal{E}$ Interfaces 2017, 9, 18748-18757.

(28) Sun, F.; He, X.; Jiang, X.; Osenberg, M.; Li, J.; Zhou, D.; Dong, K.; Hilger, A.; Zhu, X.; Gao, R.; Liu, X.; Huang, K.; Ning, D.; Markotter, H.; Zhang, L.; Wilde, F.; Cao, Y.; Winter, M.; Manke, I. Advancing Knowledge of Electrochemically Generated Lithium Microstructure and Performance Decay of Lithium Ion Battery by Synchrotron X-Ray Tomography. Materials Today 2019, 27, 21-32.

(29) Harry, K. J.; Hallinan, D. T.; Parkinson, D. Y.; MacDowell, A. A.; Balsara, N. P. Detection of Subsurface Structures Underneath Dendrites Formed on Cycled Lithium Metal Electrodes. Nature Materials 2013, 13, 69-73.

(30) Harry, K. J.; Higa, K.; Srinivasan, V.; Balsara, N. P. Influence of Electrolyte Modulus on the Local Current Density at a Dendrite Tip on a Lithium Metal Electrode. Journal of The Electrochemical Society 2016, 163, A2216-A2224.

(31) Devaux, D.; Harry, K. J.; Parkinson, D. Y.; Yuan, R.; Hallinan, D. T.; MacDowell, A. A.; Balsara, N. P. Failure Mode of Lithium Metal Batteries with a Block Copolymer Electrolyte Analyzed by X-Ray Microtomography. Journal of the Electrochemical Society 2015, 162, A1301-A1309. 
(32) Harry, K. J.; Liao, X.; Parkinson, D. Y.; Minor, A. M.; Balsara, N. P. Electrochemical Deposition and Stripping Behavior of Lithium Metal across a Rigid Block Copolymer Electrolyte Membrane. Journal of The Electrochemical Society 2015, 162, A2699A2706.

(33) Bai, P.; Li, J.; Brushett, F. R.; Bazant, M. Z. Transition of Lithium Growth Mechanisms in Liquid Electrolytes. Energy \& Environmental Science 2016, 9, 3221-3229.

(34) Rosso, M.; Gobron, T.; Brissot, C.; Chazalviel, J.-N.; Lascaud, S. Onset of Dendritic Growth in Lithium/Polymer Cells. Journal of Power Sources 2001, 97-98, 804-806.

(35) Cloetens, P.; Ludwig, W.; Boller, E.; Helfen, L.; Salvo, L.; Mache, R.; Schlenker, M. Quantitative Phase Contrast Tomography using Coherent Synchrotron Radiation. San Diego, CA, USA, 2002; pp 82-91.

(36) Peled, E. The Electrochemical Behavior of Alkali and Alkaline Earth Metals in Nonaqueous Battery Systems-The Solid Electrolyte Interphase Model. Journal of The Electrochemical Society 1979, 126, 2047-2051.

(37) Bouchet, R.; Lascaud, S.; Rosso, M. An EIS Study of the Anode Li/PEO-LiTFSI of a Li Polymer Battery. Journal of The Electrochemical Society 2003, 150, A1385-A1389.

(38) Devaux, D.; Gle, D.; Phan, T. N. T.; Gigmes, D.; Giroud, E.; Deschamps, M.; Denoyel, R.; Bouchet, R. Optimization of Block Copolymer Electrolytes for Lithium Metal Batteries. Chemistry of Materials 2015, 27, 4682-4692.

(39) Schindelin, J.; Arganda-Carreras, I.; Frise, E.; Kaynig, V.; Longair, M.; Pietzsch, T.; Preibisch, S.; Rueden, C.; Saalfeld, S.; Schmid, B.; Tinevez, J.-Y.; White, D. J.; Hartenstein, V.; Eliceiri, K.; Tomancak, P.; Cardona, A. Fiji: an Open-Source Platform for Biological-Image Analysis. Nature Methods 2012, 9, 676-682. 


\section{Graphical TOC Entry}

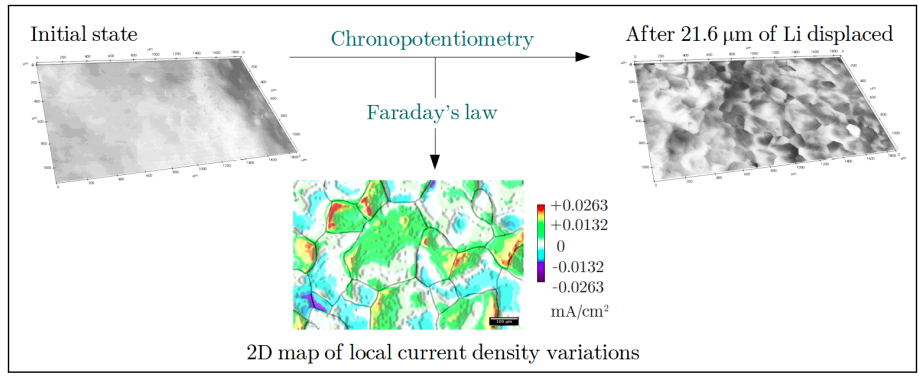

